\title{
Effects of suboptimal adherence of CPAP therapy on symptoms of obstructive sleep apnoea: a randomised, double-blind, controlled trial
}

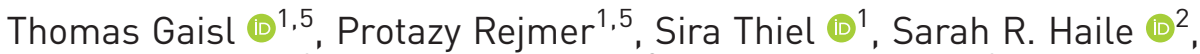

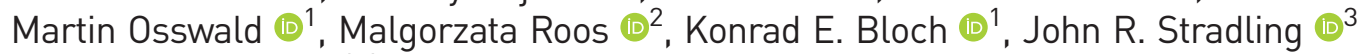 \\ and Malcolm Kohler ${ }^{1,4}$
}

@ERSpublications

Patients with obstructive sleep apnoea and daytime sleepiness are still getting a substantial benefit from suboptimal CPAP adherence (i.e. 3-4 h per night), albeit not as much as they might get if they adhered more http://bit.ly/2Phgeo2

Cite this article as: Gaisl T, Rejmer P, Thiel S, et al. Effects of suboptimal adherence of CPAP therapy on symptoms of obstructive sleep apnoea: a randomised, double-blind, controlled trial. Eur Respir J 2020; 55: 1901526 [https://doi.org/10.1183/13993003.01526-2019].

\begin{abstract}
Introduction: Continuous positive airway pressure (CPAP) is currently the treatment of choice for sleepiness in patients with obstructive sleep apnoea (OSA); however, adherence is often thought to be suboptimal. We investigated the effects of suboptimal CPAP usage on objective and subjective sleepiness parameters in patients with OSA.

Material and methods: In this 2-week, parallel, double-blind, randomised controlled trial we enrolled moderate-to-severe OSA patients with excessive pre-treatment daytime sleepiness (Epworth sleepiness scale (ESS) score $>10$ points) who had suboptimal CPAP adherence over $\geqslant 12$ months (mean nightly usage time 3-4 h). Patients were allocated through minimisation to either subtherapeutic CPAP ("sham CPAP") or continuation of CPAP ("therapeutic CPAP"). A Bayesian analysis with historical priors calculated the posterior probability of superiority.

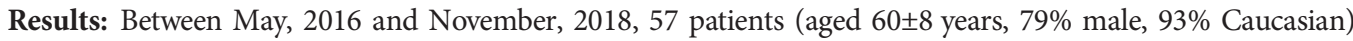
were allocated in total, and 52 who completed the study (50\% in each arm) were included in the final analysis. The unadjusted ESS score increase was 2.4 points (95\% CI $0.6-4.2, \mathrm{p}=0.01)$ in the sham-CPAP group when compared to continuing therapeutic CPAP. The probability of superiority of therapeutic CPAP over sham CPAP was $90.4 \%$ for ESS, $90.1 \%$ for systolic blood pressure and $80.3 \%$ for diastolic blood pressure.

Conclusions: Patients with moderate-to-severe OSA and daytime sleepiness are still getting a substantial benefit from suboptimal CPAP adherence, albeit not as much as they might get if they adhered more. Whether a similar statement can be made for even lower adherence levels remains to be established in future trials.
\end{abstract}

\footnotetext{
This article has an editorial commentary: https://doi.org/10.1183/13993003.00144-2020

This article has supplementary material available from erj.ersjournals.com

This trial is registered at ClinicalTrials.gov (NCT02781740).

The anonymised raw data from this trial are available upon request from the senior author.

Received: 1 Aug 2019 | Accepted after revision: 4 Dec 2019

Copyright OERS 2020
} 


\section{Introduction}

Symptomatic obstructive sleep apnoea (OSA) affects $1-2 \%$ of females and $2-4 \%$ of males in the general population, while the prevalence of asymptomatic OSA is considerably higher $[1,2]$. Untreated patients with OSA are at increased risk for all-cause and cardiovascular mortality, adverse medical outcomes and poor neurocognitive performance [3]. Patients with OSA typically complain about nonrestorative sleep, fatigue, insomnia and excessive daytime sleepiness. The most commonly mentioned reflection of the latter is that vehicle crashes are two to three times more common among patients with untreated OSA than in the general population [4].

Population-based studies have demonstrated a directly proportional relationship between the severity of OSA and healthcare costs [5]. Excessive daytime sleepiness is arbitrarily defined as an Epworth sleepiness scale (ESS) score $>10$ and applies to $23 \%$ of the general population [6]. A score $>10$ seems to independently increase healthcare utilisation [7]. Considering indirect costs of untreated OSA (e.g. due to lost productivity and illness-related accidents), its overall economic burden is likely to be far greater [8].

According to current guidelines, the treatment of choice for OSA is continuous positive airway pressure (CPAP) therapy [9]. Data from randomised controlled trials (RCTs) have consistently shown that CPAP improves sleepiness, reduces the risk of comorbidities (e.g. high blood pressure), and improves quality of life [10-12]. However, its overall effectiveness seems to broadly correlate with the average nightly usage time [11], although there is no consensus on the definition of "non-adherence". A commonly held view is that a patient should use their CPAP device for $\geqslant 4 \mathrm{~h}$ per night to experience an improvement of sleepiness and daily functioning [13-18]. However, a considerable proportion of CPAP users fail to achieve this threshold. Accordingly, 46-83\% of the patients on CPAP could be described as insufficiently treated, if optimal adherence were defined as $>4 \mathrm{~h}$ of nightly use [19].

In contrast to the findings from observational data, indirect evidence from interventional trials does not support an apparent threshold of mean usage time necessary to reduce sleepiness in OSA. A recent meta-analysis could not find a dose-dependent response of ESS to CPAP adherence, which suggests that a broad spectrum of therapy usage time is beneficial for this outcome [10]. This is in opposition to what is known from studies on comorbidities, e.g. high blood pressure, where a dose-dependent reduction could be demonstrated [11]. Thus, it remains unclear whether and to what extent suboptimally treated patients actually benefit from CPAP, or whether the treatment could be withdrawn altogether without any health-related consequences. Additionally, in some countries, reimbursement rules for CPAP devices depend on their average usage time (e.g. a threshold at $4 \mathrm{~h}$ per night); however, interventional data are lacking.

In this study, we aimed to evaluate moderate-to-severe OSA patients with documented mean CPAP usage times between 3 and $4 \mathrm{~h}$ per night for effects of therapy withdrawal on sleepiness. We hypothesised that 2 weeks of CPAP withdrawal would result in the return of OSA and its sequelae, despite apparent prior suboptimal use.

\section{Methods \\ Trial design}

This 2-week, parallel, double-blind RCT investigated the effects of suboptimal CPAP treatment on subjective sleepiness and other parameters in patients with OSA. We applied the previously described CPAP-withdrawal model $[20,21]$, where either a therapeutic or a sham CPAP device is assigned. While identical in appearance, noise production and operability, the sham CPAP device is not able to deliver a therapeutic pressure. This is achieved by 1) a built-in flow-restrictor; 2) altered software settings; and 3) a leakage at the mask-end of the tubing intended to prevent rebreathing of carbon dioxide. This design allows double-blinding and placebo effect without lessening the severity of OSA or improving the architecture of sleep [22, 23].

\section{Study population}

To be included in our RCT, patients had to 1) have a sleep-lab confirmed diagnosis of OSA with a $4 \%$ oxygen desaturation index $\left(\mathrm{ODI}_{4 \%}\right)$ of $\geqslant 15$ events. $\mathrm{h}^{-1}$ and an ESS score $>10$ points, both prior to

Affiliations: ${ }^{1}$ Dept of Pulmonology, University Hospital Zurich, Zurich, Switzerland. ${ }^{2}$ Epidemiology, Biostatistics, and Prevention Institute, University of Zurich, Zurich, Switzerland. ${ }^{3}$ National Institute for Health Research (NIHR), Oxford Biomedical Research Centre based at Oxford University Hospitals NHS Foundation Trust and University of Oxford, Oxford, UK. ${ }^{4}$ Centre for Interdisciplinary Sleep Research, University of Zurich, Zurich, Switzerland. ${ }^{5}$ Both authors contributed equally.

Correspondence: Malcolm Kohler, Chair of Respiratory Medicine, University Hospital Zurich, Rämistrasse 100, 8091 Zurich, Switzerland. E-mail: malcolm.kohlerवusz.ch 
commencing the CPAP therapy; 2) have been treated with CPAP for $\geqslant 12$ months prior to inclusion in our study, according to the 1-year statistics of the device showing a residual apnoea-hypopnea index (AHI) $<10$ events. $\mathrm{h}^{-1}$ and a mean usage time of 3-4 h per night; 3) show at least a one-time re-emergence of $\mathrm{ODI}_{4 \%} \geqslant 15$ events $\cdot \mathrm{h}^{-1}$ during a 4 -night CPAP withdrawal, as measured by ambulatory night-time pulse oximetry. The exclusion criteria were 1) previously registered ventilatory failure (awake arterial oxygen tension $<9 \mathrm{kPa}$ or arterial arterial carbon dioxide tension $>6 \mathrm{kPa}$ ); 2) unstable and/or untreated coronary or peripheral artery disease; 3) severe uncontrolled arterial hypertension (mean blood pressure values $>180 / 110 \mathrm{mmHg}$ in serial measurements); 4) previously diagnosed Cheyne-Stokes breathing pattern; 5) current professional driving; 6) age $<20$ years or $>75$ years at trial entry; 7) pregnancy. The trial was pre-registered at ClinicalTrials.gov (NCT02781740). All tests were conducted by the University Hospital Zurich (Zurich, Switzerland) and approved by the cantonal ethics committee of Zurich (KEK-ZH-Nr 2016-00332). All patients provided written informed consent according to the Declaration of Helsinki. Data were obtained according to good clinical practice guidelines.

\section{Procedures}

Visits with clinical assessments were performed at 1) inclusion; 2) baseline inpatient respiratory polygraphy upon outpatient confirmation of persistence of relevant OSA; and 3) follow-up inpatient respiratory polygraphy after 2 weeks of intervention. A detailed description of the procedures can be found in the supplementary material. The respiratory polygraphs were evaluated according to the guidelines of the American Academy of Sleep Medicine [24]. Regular controls of our RCT were performed by an external monitor who was otherwise not involved in the study.

\section{Outcomes}

The primary outcome was the change in ESS after 2 weeks. Secondary outcomes included 1) AHI and $\mathrm{ODI}_{4 \%}$ as measured by respiratory polygraphy; 2) test duration, number of missed signals and time to first four subsequently missed signals ("time to S4"), as measured by Oxford sleep resistance tests (OSLER); 3) mean/average, minimal and maximal reaction time, as measured by the multiple unprepared reaction time test (MURT); 4) mean nightly CPAP usage over 2 weeks, as protocolled by the CPAP device; 5) systolic and diastolic blood pressure and heart rate values over 2 weeks, as measured shortly before/after respiratory polygraphy and specified in the patient diary; and 6) self-assessed quality of life, as represented by parameters from the 36-item short form health survey (SF-36) and functional outcomes of sleep questionnaire (FOSQ-10).

\section{Statistical analysis}

A sample size of 52 (26 per arm) was estimated to detect the minimal clinically important difference (MCID) with $80 \%$ power at the two-sided significance level of 0.05 . This calculation assumed a standard deviation of 2.5 points for the change in ESS score between baseline and follow-up (based on our previous CPAP withdrawal trial [20]). A MCID of 2 points on the ESS was chosen based on three aspects, as follows: 1) pooled RCTs have confirmed this threshold to interpret the clinical relevance of changes in ESS [25]; 2) a 2-point decrease is considered economically viable by the National Institute for Health and Care Excellence [26]; and 3) such a decrease is expected to improve work productivity by $2 \%$ and reduce sleep-related road accidents by $\sim 9 \%$ [27].

Comparison between baseline characteristics of the intervention group were performed using the Wilcoxon rank sum test with continuity correction (continuous variables) or Fisher's exact test (categorical variables). A per-protocol analysis was performed. For all outcomes, we considered a univariate linear regression analysis adjusting for treatment group and baseline measurements of the outcome. A multivariate linear regression was performed, and a two-sided significance level of $<0.05$ was used to determine statistical significance.

In addition, we performed a Bayesian analysis because incorporation of historical data into current increases the probability of reproducibility [28]. Details on the Bayesian analysis including the systematic review can be found in the supplementary material. The statistical analysis was performed using Stata (version 15; StataCorp, College Station, TX, USA) and R (version 3.4.4; www.r-project.org).

\section{Results}

\section{Patient characteristics}

From May 27, 2016 to November 10, 2018, 1035 patients from nine Swiss sleep laboratory centres (Kantonsspital Aarau, Kantonsspital Glarus, Kantonsspital Graubünden, Spital Horgen, Spital Männedorf, Kantonsspital Schaffhausen, Stadtspital Triemli, Universitätsspital Zürich and Zürcher RehaZentrum Wald) were identified as possibly eligible and contacted by mail. 57 patients (mean \pm SD age $60.1 \pm 8.0$ years; 78.9\% male; 93\% Caucasian and 7\% Asian) were randomised. Five patients discontinued the study, one of 
them due to a hypertensive serious adverse event possibly related to the study intervention (hypertensive crisis; figure 1). The last patient completed the study on November 10, 2018. The final analysis encompassed 26 patients assigned to the sham-CPAP arm and 26 to the therapeutic-CPAP arm.

The trial profile can be seen in figure 1; the baseline characteristics in table 1. Additional information on study participants (comorbidities, medication) can be found in the supplementary material. Subtherapeutic CPAP (i.e. the sham-CPAP arm) was associated with re-emergence of OSA, as evidenced by a significant increase in AHI from baseline (+33.4 events $\cdot h^{-1}, 95 \%$ CI $23.3-43.6$ events $\left.\cdot h^{-1} ; \mathrm{p}<0.001\right)$.

\section{Primary outcome}

In comparison to the control group, sham CPAP led to an unadjusted increase in the ESS score of 2.4 points (95\% CI $0.6-4.2$ points); however, a part of this effect might have been mediated by a higher-than-baseline CPAP adherence in the control group. Although both study arms had a similar CPAP adherence during the 2 weeks prior to randomisation (mean \pm SD sham $3.5 \pm 0.6 \mathrm{~h}$, therapy $3.1 \pm 0.8 \mathrm{~h}$ ), the adherence dropped to $2.1 \pm 1.8 \mathrm{~h}$ in the sham group and rose to $4.6 \pm 1.8 \mathrm{~h}$ in the control group (66\% of all patients in this arm had a CPAP adherence $>4 \mathrm{~h}$ at follow-up). The divergence in CPAP adherence was statistically significant $(+1.9 \mathrm{~h}, 95 \%$ CI $0.4-3.6 \mathrm{~h} ; \mathrm{p}=0.017)$, but adjusting for this confirmed the statistically significance of the primary outcome (adjusted ESS score increase of +2.0 points, 95\% CI $0.5-$ 3.2 points; $\mathrm{p}=0.011$ ). Additionally, in the isolated single-arm analysis (i.e. only sham CPAP) the ESS score significantly increased by means of a paired t-test $(+2.1$ points, $95 \%$ CI $0.5-3.2 ; \mathrm{p}=0.020)$.

\section{Secondary outcomes}

Analogous to the ESS score, the FOSQ score worsened significantly in the sham intervention group (table 2). Further baseline characteristics are reported in supplementary tables S2 and S3. The results of all secondary outcomes can be found in table 2 and supplementary table S4.

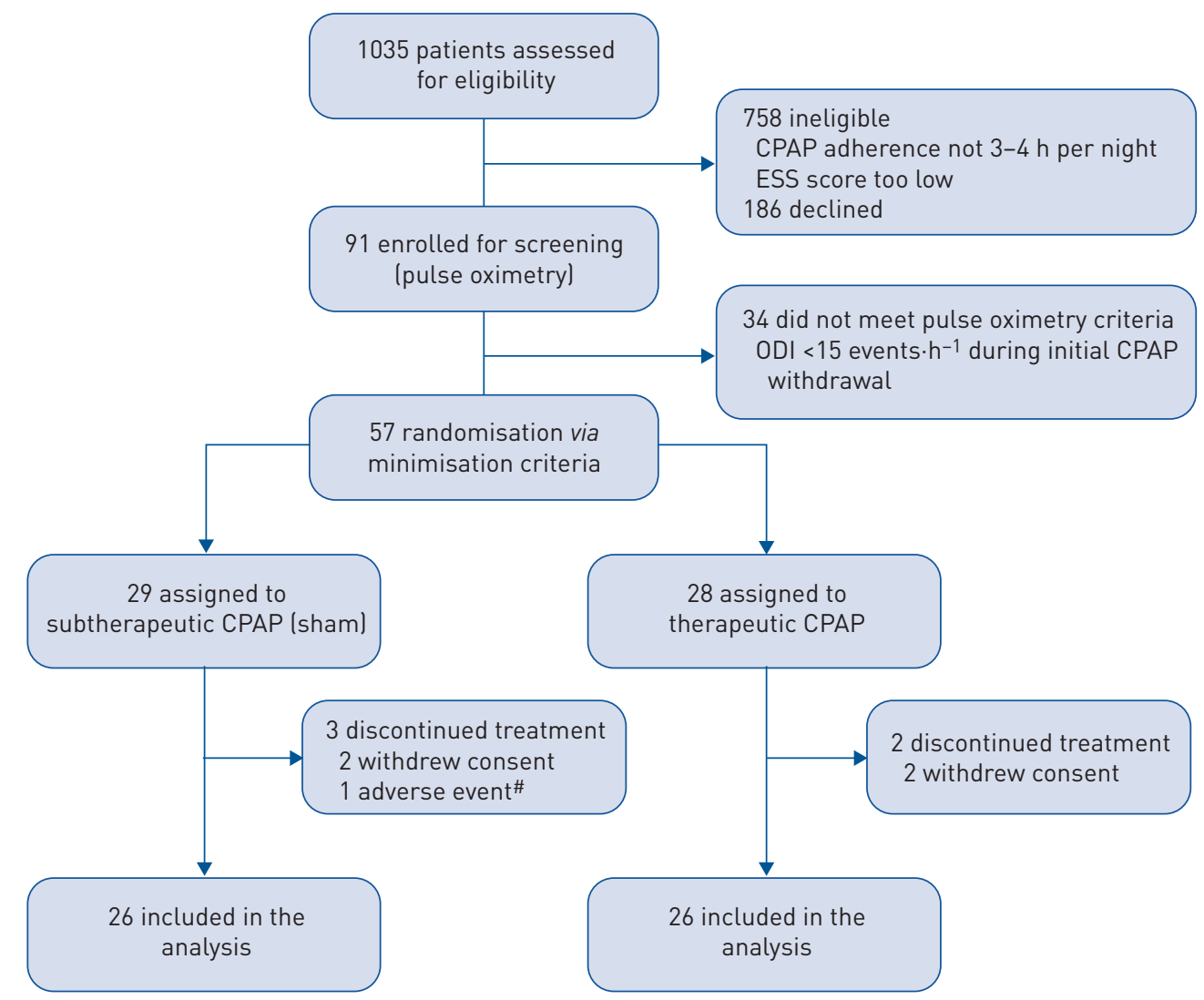

FIGURE 1 Trial profile. " : one patient was unblinded and consequently withdrawn from the trial due to a hypertensive emergency. CPAP: continuous positive airway pressure; ESS: Epworth sleepiness scale; ODI: oxygen desaturation index. 
TABLE 1 Baseline characteristics of the intention-to-treat population

Subtherapeutic CPAP (sham)

Therapeutic CPAP (real)

\begin{tabular}{|c|c|c|}
\hline Subjects & 29 & 28 \\
\hline \multicolumn{3}{|l|}{ Anthropometrics } \\
\hline Age years & $61.5 \pm 6.5$ & $60.1 \pm 8.7$ \\
\hline Male & $21(81)$ & $21(81)$ \\
\hline $\mathrm{BMI} \mathrm{kg} \cdot \mathrm{m}^{-2}$ & $32.2 \pm 4.4$ & $33.0 \pm 4.9$ \\
\hline Height $\mathrm{cm}$ & $172.1 \pm 9.7$ & $174.6 \pm 7.5$ \\
\hline Weight kg & $95.6 \pm 16.0$ & $100.1 \pm 13.3$ \\
\hline Neck circumference $\mathrm{cm}$ & $42.2 \pm 3.6$ & $43.5 \pm 3.5$ \\
\hline Waist circumference $\mathrm{cm}$ & $113 \pm 14$ & $115 \pm 14$ \\
\hline Hip circumference cm & $108 \pm 11$ & $108 \pm 11$ \\
\hline Mallampati score class & $2.7 \pm 1.0$ & $2.4 \pm 1.0$ \\
\hline \multicolumn{3}{|l|}{ CPAP adherence data } \\
\hline CPAP adherence (over 365 days) h.day ${ }^{-1 \#}$ & $3.5 \pm 0.4$ & $3.3 \pm 0.4$ \\
\hline Leakage (over 365 days) L.min ${ }^{-1 \#}$ & $1(0-6)$ & $0(0-2.5)$ \\
\hline \multicolumn{3}{|l|}{ Obstructive sleep apnoea } \\
\hline Time since diagnosis of OSA years & $5.4 \pm 3.3$ & $5.4 \pm 3.2$ \\
\hline $\mathrm{AHI}$ at diagnosis events $\cdot \mathrm{h}^{-1}$ & $46.7 \pm 21.8$ & $38.8 \pm 17.9$ \\
\hline AHI during CPAP events $\cdot h^{-1 \#}$ & $3.3 \pm 2.5$ & $2.2 \pm 2.5$ \\
\hline ESS at diagnosis points & $12.3 \pm 2.6$ & $12.1 \pm 2.4$ \\
\hline ESS at study inclusion points" & $8.5 \pm 3.9$ & $9.5 \pm 4.7$ \\
\hline $\mathrm{AHI}$ during CPAP events $\cdot \mathrm{h}^{-1 \#}$ & $3.3 \pm 2.5$ & $2.2 \pm 2.5$ \\
\hline
\end{tabular}

Data are presented as $\mathrm{n}$, mean $\pm \mathrm{SD}, \mathrm{n}(\%)$ or median (interquartile range). CPAP: continuous positive airway pressure; BMI: body mass index; OSA: obstructive sleep apnoea; ASI: apnoea-hypopnoea index; ESS: Epworth sleepiness scale. ${ }^{\#}$ : data downloaded from CPAP device; ${ }^{\text {I: }}$ before pulse oximetry.

Objective sleepiness parameters

There was no effect on either of the independent ways to objectively assess sleepiness (i.e. the OSLER and MURT tests). A nonsignificant trend which corresponded to the ESS change was mostly limited by a low sample size (table 2).

\section{Usage patterns}

$28(49 \%)$ participants reported that their pre-trial suboptimal CPAP adherence was due to their lifestyle, $25(44 \%)$ due to comorbidities and four (7\%) due to "technical issues". Further information regarding the categorisation and the individual reasons given can be found in supplementary table S5. CPAP adherence patterns did not differ between these three groups ( $\mathrm{p}$-value for difference (global test $)=0.523$ ).

\section{Bayesian analysis}

Based on a systematic review (supplementary figure S1), historical data from four trials were included for ESS (2866 patients) [29-32], while only two provided historical data for blood pressure (2376 patients) $[29,30]$. In the current study data, we observed a difference in ESS between the treatment arms of $\sim 2.4$ points, while in the historical data there was a mean difference of $\sim 1.3$ points. In addition, small differences in blood pressure were observed in the current study data (table 2), and in the historical data (systolic blood pressure: sham group $+1.3 \mathrm{mmHg}$, therapeutic group $-0.01 \mathrm{mmHg}$; diastolic blood pressure: sham group $-0.1 \mathrm{mmHg}$, therapeutic group $-0.7 \mathrm{mmHg}$ ). Sampling from the posterior distributions and computing the differences between the treatment arms, we obtained a median difference in delta ESS of 0.825 points (sham - therapeutic, positive favours therapeutic; $95 \%$ credible interval -0.41 2.05 points). The posterior probability of superiority for ESS and blood pressure is $90.4 \%$ (table 3 ).

\section{Discussion}

This is the first RCT to investigate explicitly predefined suboptimal CPAP usage and delineate individually reported reasons for lower adherence in a representative population. Our RCT demonstrated that suboptimal adherence to CPAP therapy improves subjective daytime sleepiness in patients with moderate-to-severe OSA, since withdrawal of therapeutic CPAP for 2 weeks resulted in a +2.4 -point increase or $90.4 \%$ probability of superiority on the ESS. Large-scale meta-analyses in this field have suggested that CPAP use in similar populations is associated with an even greater reduction in ESS score of 2.5-2.9 points $[10,33$. The effect of suboptimal CPAP therapy on subjective daytime sleepiness was 
TABLE 2 Per-protocol analysis on primary and secondary outcomes

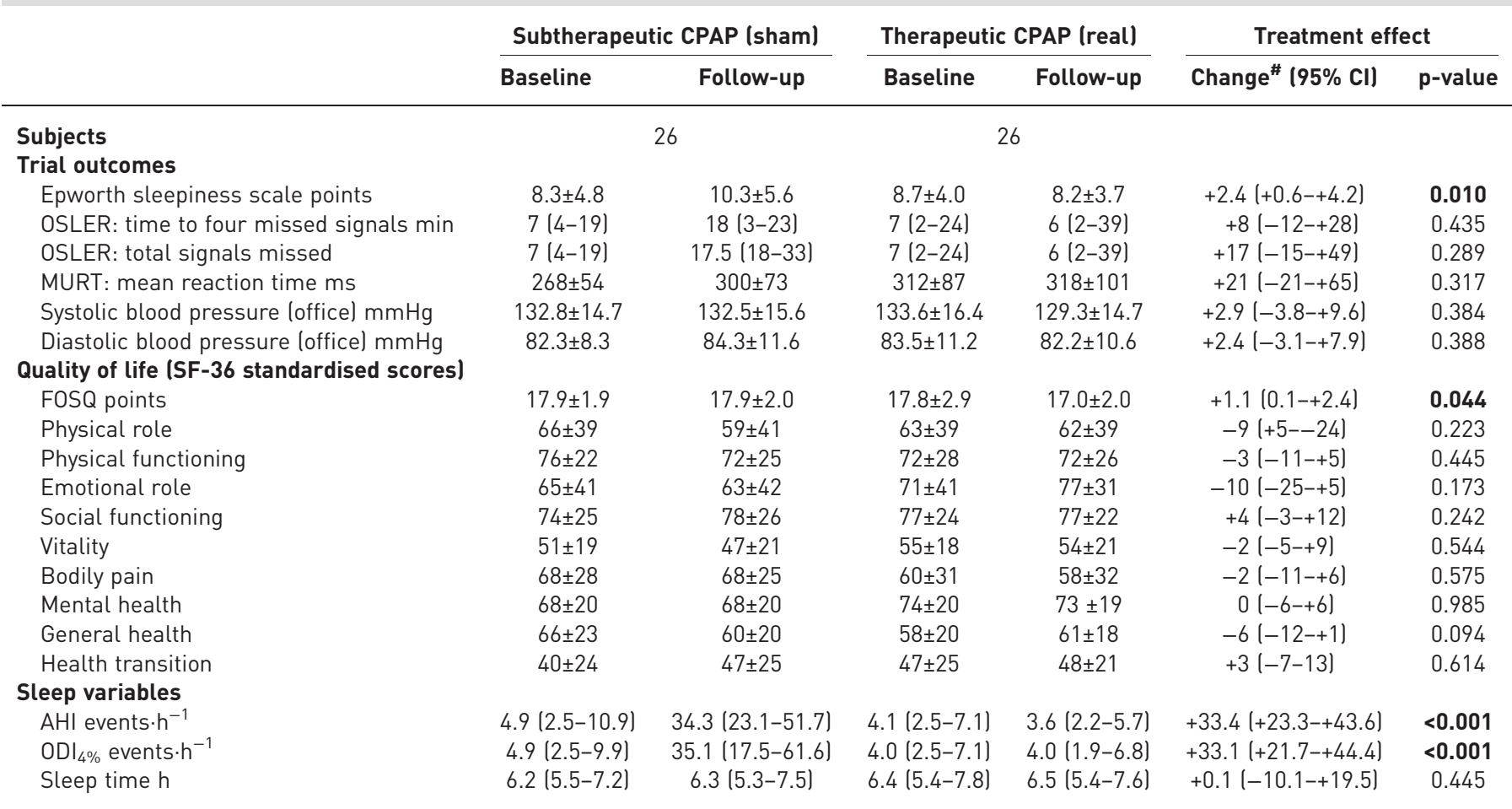

Data are presented as $\mathrm{n}$, mean \pm SD or median (interquartile range), unless otherwise stated. Bold type represents statistical significance. CPAP: continuous positive airway pressure; OSLER: Oxford sleep resistance test; MURT: multiple unprepared reaction time test; SF-36: short-form 36-item questionnaire; FOSQ: functional outcomes of sleep questionnaire 10; $\mathrm{AHI}$ : apnoea-hypopnoea index; $0 \mathrm{DI}_{4 \%}$ : oxygen desaturation index. \#: adjusted for baseline: in the isolated single-arm analysis the primary outcome significantly increased by +2.2 points $195 \% \mathrm{Cl} 0.7-3.8$; $\mathrm{p}=0.005$ ) in the sham CPAP arm.

\begin{abstract}
virtually the same as that reported for usage times of 4-7 h per day [10]. However, approximately a third of our effect size might be due to improvements due to increases in CPAP adherence (Results section). Whether similar statements can be made for lower adherence remains to be established in future trials. In other words, this trial does not foreclose a non-existing dose-response effect of CPAP on subjective daytime sleepiness; the potential threshold might just be lower than $3 \mathrm{~h}$ of usage time.

The effect of CPAP on the ESS score in our trial (per-protocol analysis +2.4 points, adjusted: +2.0 points; single-arm analysis +2.1 points) was robust and confirmed our primary hypothesis $(+2$ points). Previous studies confirm that this 2-point change is not only statistically significant, but also clinically relevant [25-27, 34]. Conversely, we were not able find objectively measurable correlates of sleepiness (i.e. significant effects for the OSLER and MURT tests). Correspondingly, a previous meta-analysis indicated that the effect of CPAP on subjective sleepiness is generally larger than on objective measurements [33], which could provide partial explanation for our results. Since objective measures have always been less sensitive compared to subjective ones in this setting, one can hypothesise that objective measures (e.g. the OSLER or MURT tests) do not fully characterise the symptoms of a patient.
\end{abstract}

TABLE 3 Primary and secondary end-point Bayesian analysis using historical data from four randomised controlled trials (RCTs) [29-32] with a continuous positive airway pressure adherence of 3-4 $\mathrm{h}$

Sample size ${ }^{\#}$ Lower boundary

\begin{tabular}{|c|c|c|c|c|c|c|}
\hline Epworth sleepiness scale & $52+2866$ & -0.4128 & 0.8248 & 2.051 & 0.0009 & 90.41 \\
\hline Systolic blood pressure & $52+2376$ & -0.8241 & 1.6045 & 4.043 & 0.0009 & 90.17 \\
\hline Diastolic blood pressure & $52+2376$ & -1.0352 & 0.8044 & 2.662 & 0.0013 & 80.27 \\
\hline
\end{tabular}

A detailed description of the analysis and the systematic review behind this method can be found in the supplementary material. ${ }^{\#}$ : current data+historical priors (four RCTs [29-32]). 
In our study, participants of both arms diverged from their previously similar mean nightly CPAP times (therapy $3.1 \pm 0.8 \mathrm{~h}$, sham $3.5 \pm 0.6 \mathrm{~h}$ ) towards higher values in the therapeutic group $(4.6 \pm 1.8 \mathrm{~h}$ ) and lower values in the sham group $(2.1 \pm 1.8 \mathrm{~h})$. This yielded a significant mean difference of $1.9 \mathrm{~h}$. The substantial impact of allocation to therapy adherence has already been reported in other interventional trials by other groups involving sham CPAP devices and seems to be a universal phenomenon across sex, race and age boundaries [23, 35-37]. In the literature, this phenomenon is also referred to as the "Hawthorne effect", where individuals modify an aspect of their behaviour in response to their awareness of being observed. Interestingly, in multivariable analyses, the treatment allocation was the strongest predictor of CPAP adherence within RCTs [35]. Possible additional explanations for this include (subconscious) dissatisfaction with the current treatment in the sham CPAP arm, or simply unblinding [36]. Indeed, data from a meta-analysis suggests that a clinically significant proportion $(\sim 30 \%)$ of the effectiveness of CPAP adherence in reducing sleepiness is probably caused by patient expectation of benefit [38]. In our study, we could rule out substantial unblinding, as only $59 \%$ of all study participants correctly guessed their allocation. This is in line with previous results by other groups using the CPAP withdrawal model, where $56 \%$ of participants could guess their true allocations correctly, which was only slightly higher than could be expected by chance [23]. The fact that the single-arm analysis (i.e. only sham CPAP) also showed a clinically significant effect on the ESS is reassuring. We suspect that the patients from the suboptimal collective might be more sensitive to any changes in their therapy regimens, for example on account of lifestyle circumstances and comorbidities having been classified as $>90 \%$ of the reasons reported behind their generally lower adherence (supplementary table S5). Considering other potential benefits of longer nightly CPAP usage (e.g. lower arterial blood pressure [11]), future trials should investigate different, focused ways of boosting adherence that might be efficiently incorporated in the clinical setting.

Although previous studies did not show a dose-dependent reaction of many objective surrogates of daytime sleepiness (e.g. maintenance of wakefulness test) to CPAP adherence, they were able to detect significant drops in proportions of patients with normalised or significantly improved ESS-, FOSQ- and SF-36 scores when CPAP was used $<4 \mathrm{~h}$ per night $[13,15,17,18]$. Already-compliant patients were also demonstrated to increase their mean usage times and relevant scores even further upon additional, intensive support [39]. These notions most probably led to the widely recommended threshold of $4 \mathrm{~h}$ of mean usage time of CPAP therapy to be considered sufficient. Still, the meta-analysis comparing treatment effects of CPAP versus mandibular advancement devices, encompassing 67 studies, noted no evidence of studies reporting higher CPAP adherence also reporting larger treatment effects $(p=0.7)$ [10].

In lieu of our findings and pre-existing literature, the widely used 4-h threshold for clinical benefit seems unjustified. We could think of several additional reasons to support that claim, as follows. 1) Sleep and sleepiness feature a great interpersonal variability, and a one-size-fits-all approach may not be adequate. 2) There seems to be a substantial subgroup of patients with OSA who, despite sleeping for $>5 \mathrm{~h}$ per day, deliberately keep their CPAP intervals on levels which might be regarded as "suboptimal" (i.e. $<4 \mathrm{~h}$ per day) over a long period of time. When asked about their motivation, these patients often report being satisfactory treated at "their personal level." 3) Sleep itself is not uniform in its function and does exhibit a substantial variation in terms of restorability. CPAP therapy might therefore indeed have a lower time threshold for countering excessive sleepiness, which would not necessarily be applicable to countering other sequelae of OSA (e.g. high blood pressure). Apart from the possibility of the overall time thresholds for countering sleepiness and other sequelae being different, it is conceivable that other aspects play their roles as well. The thresholds of CPAP efficacy on different sequelae of OSA may not only be individual, but also composite, e.g. involving patterns of usage, adaptive pressures or varying air compositions.

The current trial population only consisted of a selected group of patients with excessive daytime sleepiness and moderate-to-severe OSA, thus the conclusions might not be generalisable to other populations with OSA. Another limitation of the current trial is the relatively short withdrawal period of 2 weeks, which might not feature the full effect of CPAP on daytime sleepiness. However, the ESS was primarily designed for a 2 -week period and the test-retest reliability in this timeframe is sufficiently high [40]. Finally, the ESS score itself is prone to subjectivity and does represent an ordinal variable (and not an interval scale), thus conclusions regarding the effect size might be distorted [41]. Additionally, potential unblinding might have contributed to CPAP adherence during the trial and thus affected the outcome. Nevertheless, the ESS score was chosen as the primary outcome, as it is the most widely used clinical instrument for evaluating sleepiness and most investigated marker for subjective daytime sleepiness.

We conclude, that patients with daytime sleepiness are still getting a substantial benefit from suboptimal CPAP adherence, albeit not as much as they might get if they adhered more. Therefore, suboptimal CPAP usage between 3 and $4 \mathrm{~h}$ per night in moderate-to-severe patients with OSA might not be a valid reason to stop treatment or not reimburse treatment at all. Whether a similar statement can be made lower adherence and/or very severe OSA remains to be established in future trials. Therefore, future trials should 
consider a larger spectrum of CPAP usage patterns (different mean usage times and their intervalled distributions) in a variety of OSA patients. Attention should be drawn to investigating different, focused ways of boosting adherence that might be efficiently incorporated in the clinical setting.

Acknowledgements: The authors would like to thank all colleagues who were involved in the recruitment process, especially Tsogyal Latshang (Kantonsspital Graubünden), Sarosh Irani (Kantonsspital Aarau), Yvonne Nussbuamer (Kantonsspital Schaffhausen), Robert Thurnheer (Kantonsspital Münsterlingen), Jean-Luc Kurzen (Spital Männedorf), Marc Spielmanns (Zürcher RehaZentrum Wald), Alexander Turk and Patrick Schihin (Spital Horgen), Thomas Brack and Alice Odermatt (Lungenliga Glarus) and Michael Schlunegger, Dominic Karrer and Sylke Meier (Lunge Zürich). We also thank the patients who committed to the study and often travelled long distances to participate. Without their effort, this project would not have been possible.

Author contributions: T. Gaisl, M. Roos and S.R. Haile had full access to all of the data in the study and take responsibility for the integrity of the data and the accuracy of the data analysis. Study concept and design: T. Gaisl, K.E. Bloch, J.R. Stradling and M. Kohler. Acquisition, analysis or interpretation of data: all authors. Drafting of the manuscript: T. Gaisl. Critical revision of the manuscript for important intellectual content: all authors. Statistical analysis: T. Gaisl, M. Roos and S.R. Haile. Administrative, technical or material support: T. Gaisl and M. Kohler. Study supervision: M. Kohler.

Support statement: This was an investigator-initiated trial and supported by the Swiss National Science Foundation (project number 32003B_162534). The funder of the study had no role in study design, data collection, data analysis, data interpretation, or writing of the report. Funding information for this article has been deposited with the Crossref Funder Registry.

Conflict of interest: P. Rejmer has nothing to disclose. S. Thiel has nothing to disclose. S.R. Haile has nothing to disclose. M. Osswald has nothing to disclose. M. Roos has nothing to disclose. K.E. Bloch has nothing to disclose. J.R. Stradling reports personal fees for consultancy from Bayer AG and ResMed, outside the submitted work. M. Kohler reports personal fees from Bayer AG, grants from University of Zurich and Lunge Zurich, outside the submitted work. T. Gaisl reports personal fees for consultancy from Bayer AG, outside the submitted work.

\section{References}

1 Young T, Peppard PE, Gottlieb DJ. Epidemiology of obstructive sleep apnea: a population health perspective. Am J Respir Crit Care Med 2002; 165: 1217-1239.

2 Heinzer R, Vat S, Marques-Vidal P, et al. Prevalence of sleep-disordered breathing in the general population: the HypnoLaus study. Lancet Respir Med 2015; 3: 310-318.

3 Molnar MZ, Mucsi I, Novak M, et al. Association of incident obstructive sleep apnoea with outcomes in a large cohort of US veterans. Thorax 2015; 70: 888-895.

4 George CF. Sleep apnea, alertness, and motor vehicle crashes. Am J Respir Crit Care Med 2007; 176: 954-956.

5 Kapur V, Blough DK, Sandblom RE, et al. The medical cost of undiagnosed sleep apnea. Sleep 1999; 22: 749-755.

6 Sander C, Hegerl U, Wirkner K, et al. Normative values of the Epworth Sleepiness Scale (ESS), derived from a large German sample. Sleep Breath 2016; 20: 1337-1345.

7 Ronksley PE, Hemmelgarn BR, Heitman SJ, et al. Excessive daytime sleepiness is associated with increased health care utilization among patients referred for assessment of OSA. Sleep 2011; 34: 363-370.

8 Sassani A, Findley LJ, Kryger M, et al. Reducing motor-vehicle collisions, costs, and fatalities by treating obstructive sleep apnea syndrome. Sleep 2004; 27: 453-458.

9 Epstein LJ, Kristo D, Strollo PJ, Jr, et al. Clinical guideline for the evaluation, management and long-term care of obstructive sleep apnea in adults. J Clin Sleep Med 2009; 5: 263-276.

10 Bratton DJ, Gaisl T, Schlatzer C, et al. Comparison of the effects of continuous positive airway pressure and mandibular advancement devices on sleepiness in patients with obstructive sleep apnoea: a network meta-analysis. Lancet Respir Med 2015; 3: 869-878.

11 Bratton DJ, Gaisl T, Wons AM, et al. CPAP vs mandibular advancement devices and blood pressure in patients with obstructive sleep apnea: a systematic review and meta-analysis. JAMA 2015; 314: 2280-2293.

12 Kuhn E, Schwarz EI, Bratton DJ, et al. Effects of CPAP and mandibular advancement devices on health-related quality of life in OSA: a systematic review and meta-analysis. Chest 2017; 151: 786-794.

13 Antic NA, Catcheside P, Buchan C, et al. The effect of CPAP in normalizing daytime sleepiness, quality of life, and neurocognitive function in patients with moderate to severe OSA. Sleep 2011;34: 111-119.

14 Sawyer AM, Gooneratne NS, Marcus CL, et al. A systematic review of CPAP adherence across age groups: clinical and empiric insights for developing CPAP adherence interventions. Sleep Med Rev 2011; 15: 343-356.

15 Weaver TE, Maislin G, Dinges DF, et al. Relationship between hours of CPAP use and achieving normal levels of sleepiness and daily functioning. Sleep 2007; 30: 711-719.

16 Zimmerman ME, Arnedt JT, Stanchina M, et al. Normalization of memory performance and positive airway pressure adherence in memory-impaired patients with obstructive sleep apnea. Chest 2006; 130: 1772-1778.

17 Weaver T. Adherence with Continuous Positive Airway Pressure (CPAP). UpToDate 2018. www.uptodate.com/ contents/adherence-with-continuous-positive-airway-pressure-cpap Date last accessed: December 18, 2018. Date last updated: October 9, 2019.

18 Masa JF, Corral-Peñafiel J. Should use of 4 hours continuous positive airway pressure per night be considered acceptable compliance? Eur Respir J 2014; 44: 1119-1120.

19 Weaver TE, Grunstein RR. Adherence to continuous positive airway pressure therapy: the challenge to effective treatment. Proc Am Thorac Soc 2008; 5: 173-178.

20 Kohler M, Stoewhas AC, Ayers L, et al. Effects of continuous positive airway pressure therapy withdrawal in patients with obstructive sleep apnea: a randomized controlled trial. Am J Respir Crit Care Med 2011; 184: 1192-1199. 
21 Schwarz EI, Martinez-Lozano Sinues P, Bregy L, et al. Effects of CPAP therapy withdrawal on exhaled breath pattern in obstructive sleep apnoea. Thorax 2016; 71: 110-117.

22 Schwarz EI, Schlatzer C, Stehli J, et al. Effect of CPAP withdrawal on myocardial perfusion in OSA: a randomized controlled trial. Respirology 2016; 21: 1126-1133.

23 Chasens ER, Drumheller OJ, Strollo PJ Jr. Success in blinding to group assignment with sham-CPAP. Biol Res Nurs 2013; 15: 465-469.

24 Berry RB, Budhiraja R, Gottlieb DJ, et al. Rules for scoring respiratory events in sleep: update of the 2007 AASM Manual for the Scoring of Sleep and Associated Events. Deliberations of the Sleep Apnea Definitions Task Force of the American Academy of Sleep Medicine. J Clin Sleep Med 2012; 8: 597-619.

25 Crook S, Sievi NA, Bloch KE, et al. Minimum important difference of the Epworth Sleepiness Scale in obstructive sleep apnoea: estimation from three randomised controlled trials. Thorax 2019; 74: 390-396.

26 Weatherly HL, Griffin SC, Mc Daid C, et al. An economic analysis of continuous positive airway pressure for the treatment of obstructive sleep apnea-hypopnea syndrome. Int J Technol Assess Health Care 2009; 25: 26-34.

27 Craig SE, Kohler M, Nicoll D, et al. Continuous positive airway pressure improves sleepiness but not calculated vascular risk in patients with minimally symptomatic obstructive sleep apnoea: the MOSAIC randomised controlled trial. Thorax 2012; 67: 1090-1096.

28 McShane BB, Böckenholt U. Single-paper meta-analysis: benefits for study summary, theory testing, and replicability. J Consum Res 2017; 43: 1048-1063.

29 Hoyos CM, Killick R, Yee BJ, et al. Cardiometabolic changes after continuous positive airway pressure for obstructive sleep apnoea: a randomised sham-controlled study. Thorax 2012; 67: 1081-1089.

30 McEvoy RD, Antic NA, Heeley E, et al. CPAP for prevention of cardiovascular events in obstructive sleep apnea. N Engl J Med 2016; 375: 919-931.

31 Redline S, Adams N, Strauss ME, et al. Improvement of mild sleep-disordered breathing with CPAP compared with conservative therapy. Am J Respir Crit Care Med 1998; 157: 858-865.

32 Weaver TE, Mancini C, Maislin G, et al. Continuous positive airway pressure treatment of sleepy patients with milder obstructive sleep apnea: results of the CPAP Apnea Trial North American Program (CATNAP) randomized clinical trial. Am J Respir Crit Care Med 2012; 186: 677-683.

33 Patel SR, White DP, Malhotra A, et al. Continuous positive airway pressure therapy for treating sleepiness in a diverse population with obstructive sleep apnea: results of a meta-analysis. Arch Intern Med 2003; 163: 565-571.

34 Patel S, Kon SSC, Nolan CM, et al. The Epworth sleepiness scale: minimum clinically important difference in obstructive sleep apnea. Am J Respir Crit Care Med 2018; 197: 961-963.

35 May AM, Gharibeh T, Wang L, et al. CPAP adherence predictors in a randomized trial of moderate-to-severe OSA enriched with women and minorities. Chest 2018; 154: 567-578.

36 Budhiraja R, Kushida CA, Nichols DA, et al. Impact of randomization, clinic visits, and medical and psychiatric cormorbidities on continuous positive airway pressure adherence in obstructive sleep apnea. J Clin Sleep Med 2016; 12: 333-341.

37 Paz Y Mar HL, Hazen SL, Tracy RP, et al. Effect of continuous positive airway pressure on cardiovascular biomarkers: the Sleep Apnea Stress Randomized Controlled Trial. Chest 2016; 150: 80-90.

38 Crawford MR, Bartlett DJ, Coughlin SR, et al. The effect of continuous positive airway pressure usage on sleepiness in obstructive sleep apnoea: real effects or expectation of benefit? Thorax 2012; 67: 920-924.

39 Bouloukaki I, Giannadaki K, Mermigkis C, et al. Intensive versus standard follow-up to improve continuous positive airway pressure compliance. Eur Respir J 2014; 44: 1262-1274.

40 Johns MW. Reliability and factor analysis of the Epworth Sleepiness Scale. Sleep 1992; 15: 376-381.

41 Campbell AJ, Neill AM, Scott DAR. Clinical reproducibility of the Epworth sleepiness scale for patients with suspected sleep apnea. J Clin Sleep Med 2018; 14: 791-795. 